\title{
Crossover of interfacial dynamics
}

\author{
David Jasnow \\ Department of Physics and Astronomy, University of Pittsburgh, Pittsburgh, Pennsylvania 15260 \\ R. K. P. Zia \\ Physics Department, Virginia Polytechnic Institute and State University, Blacksburg, Virginia 24061
}

(Received 15 April 1987)

\begin{abstract}
Some dynamical models in which there is a significant interplay between interfacial and bulk degrees of freedom are treated at the mean-field (or Van Hove) level from a coarse-grained microscopic viewpoint. Specifically, the near-equilibrium interfacial dynamics of two of the simplest models with conservation laws, models $B$ and $C$, are studied with use of (as appropriate) variational techniques, perturbation theory, and (with certain additional simplifications) exact solutions. Use of these methods allows the dispersion relation for interfacial modes to be interpolated between the "hydrodynamical" and critical regimes. The crossover scaling behavior lends support to renormalizationgroup methods near $d=1$ which focus on the interfacial modes but nonetheless extract the bulk dynamical exponent as well as the crossover.
\end{abstract}

\section{INTRODUCTION}

The behavior of interfaces, regions separating coexisting phases, has been of great interest since Gibbs' treatise. ${ }^{1}$ Several recent reviews of equilibrium properties are available ${ }^{2-4}$ which document the considerable progress made in the field over the past few years. Studies of the dynamical aspects of interfacial phenomena are also extensive, appearing in a variety of topics, from hydrodynamics ${ }^{5-8}$ to metallurgy, ${ }^{9-13}$ from equations linearized near equilibrium to systems driven far from equilibrium. ${ }^{14}$ More recently, field-theoretic investigations of the dynamics of systems near criticality were begun, ${ }^{15-17}$ and connections between the bulk and interface were explored. ${ }^{18-20}$ Much remains to be learned about this regime, in which the bulk has its own interesting properties and there is a significant interplay between interfacial and bulk degrees of freedom. Such a regime must be treated from a more microscopic viewpoint, starting from a bulk Hamiltonian rather than just an interface model. Information garnered from such studies should prove important in the development of effective interfacial models for more complex systems such as those driven far from equilibrium.

The prototypical case of a system in which there is an interplay between bulk and interfacial degrees of freedom is that of a system of two coexisting phases not too far from criticality. In the limit in which interfacial distortions are of long wavelength compared with any thermal (correlation) lengths in the problem, the description of the interfacial modes should go over to a hydrodynamic description. In the opposite limit of long correlation lengths, the bulk criticality becomes important, and there is a strong connection between bulk and interfacial degrees of freedom. The dynamical (and static) interfacial properties show a crossover from hydrodynamic to critical. From a slightly more fundamental viewpoint one has the following description. The fluctuations of the inter- face in an Ising-like system are governed by soft, Goldstone modes arising from the broken translational invariance in the establishment of the interface itself. The interfacial fluctuations arising from these modes contain the necessary information about criticality for the ultimate disappearance of the interface itself.

The program one would like to follow is to begin with a bulk Hamiltonian and establish an interface with the use of appropriate boundary conditions (or wall potentials) and thermodynamic parameters. Interfacial static and dynamic properties, at least near equilibrium, should follow from the response properties of the full inhomogeneous system. For real fluid systems, in which there is a coupling of the order parameter to velocity fields, this represents a difficult problem. Felderhof ${ }^{7}$ and Turski and Langer ${ }^{8}$ made some progress in this direction by treating the statistical mechanics of the fluid within a mean-field approach and treating the fluid as inviscid. The calculations amount to the hydrodynamic treatment of a diffuse interface, but even within the approximations noted they are not without difficulties.

It has been possible to go beyond the mean-field treatment of the thermal properties only in the simplest possible case $\mathrm{e}^{15-17}$ in which velocity fields are entirely neglected (corresponding to a solid), and the order parameter is taken as nonconserved, corresponding to an order-disorder system. For such a system [model $A$ (Ref. 21 )] the interfacial dispersion relation could be extracted from the response of the system; the dispersion relation has the form of a scaling function

$$
-\iota \omega_{q}=q^{z} \Omega(q \xi),
$$

where $q$ is the $(d-1)$-dimensional wave vector in the plane of the interface and $\xi$ is the bulk correlation length. The exponent $z$ is a dynamic exponent which one expects may be identified with the dynamic scaling exponent for the bulk homogeneous system. As $q \xi \rightarrow \infty$, that is, as the 
critical regime is approached, the interface disappears and there should be one characteristic frequency $\omega_{q} \sim q^{z} .^{21}$ In the opposite limit, the hydrodynamic regime characterized by $q \xi \rightarrow 0$, one expects $\omega_{q} \sim q^{\sigma}$, with $\sigma$ typically an integer or simple fraction. The scaling function $\Omega(x)$ must develop a singularity as $x \rightarrow 0$ in order to go over to this new behavior. The correlation and response properties must also exhibit crossover behavior between the two limiting regimes. The renormalization-group treatment of model- $\boldsymbol{A}$ interfacial dynamics near $d=4$ confirms this picture, ${ }^{16,17}$ but a two-loop calculation of the dynamics of the inhomogeneous system is required to probe the full structure. The scaling picture lends support to the renormalization-group treatment near $d=1,{ }^{15}$ which calculates $z$ (the bulk exponent), as well as the full crossover from the critical to the hydrodynamic regime by focusing on interfacial properties alone.

The purely relaxational behavior of model $A$ is too simple to describe the dynamical behavior of many physical systems of interest. One must be able to include conservation laws, fluid velocity fields and "hydrodynamical" interactions, and possible mode coupling terms to describe more interesting dynamics of physical systems. Given the complexity of including fluctuations in the interfacial dynamics of model $A$ (Refs. 15-17), extending the treatment to include these additions will be extremely difficult. One is forced to turn to mean-field (zero-loop or Van Hove level) dynamics; fortunately, interesting crossover behavior already occurs at this level as soon as conservation laws are introduced.

In this short paper we consider the near-equilibrium interfacial dynamics of two of the simplest models with conservation laws, the so-called models $B$ and $C .{ }^{21}$ By the use of a variational principle the dispersion relations for the interfacial modes of these models can be interpolated between the critical and hydrodynamical regimes. We also comment on the connection between the variational approach and some interfacial equations of motion recently discussed by Ohta and Kawasaki. ${ }^{18-20}$ The layout of this paper is as follows. In Sec. II the case of conserved order parameter (model $B$ ) is briefly treated while in Sec. III the case of a conserved auxiliary field coupling to a nonconserved order parameter (model $C$ ) is considered. In Sec. IV some brief concluding remarks are made.

\section{CONSERVED ORDER PARAMETER}

We consider systems whose coarse-grained dynamics are described by the time-dependent Ginsburg-Landau equation for the evolution of the local order parameter $\phi(x)$. The time dependence is assumed to follow from

$$
\partial_{t} \phi=\Gamma \nabla^{2}(\delta H / \delta \phi),
$$

where $H$ is the usual Ginzburg-Landau Hamiltonian (in a form useful for considering $T<T_{c}$ ),

$$
\begin{aligned}
& H=\int\left[\frac{1}{2}(\nabla \phi)^{2}+U(\phi)\right] d^{d} x, \\
& U(\phi)=\frac{1}{2}\left(\phi^{2}-M^{2}\right)^{2},
\end{aligned}
$$

and $\Gamma$ is the kinetic coefficient, which can be set to unity by an appropriate choice of units. The miscibility gap is $2 M$ in this notation and the bulk correlation length is given by $\xi^{-1}=2 M$. The equilibrium interface is found, at this level, from solutions of $\delta H / \delta \phi=0$ subject to appropriate (odd) boundary conditions. The equilibrium interface (classical ground state) is the familiar $\phi=\phi_{c}(z) \propto \tanh (z / 2 \xi)$, where $z$ is the direction perpendicular to the plane of the interface. (Relevant background can be found in Refs. 3 and 4.) When the equation of motion (2.1) is linearized about the flat equilibrium interface given by the classical solution $\phi_{c}$, so that $\phi=\phi_{c}+\psi$, one finds that interfacial modes of wave number $q$ (in the plane of the interface) have complex frequency $\omega$ determined from

$$
(-i \omega) \psi=D(-D+V) \psi,
$$

where $D \equiv \mathrm{\partial}_{z}^{2}-q^{2}$ and $L_{z}=-D+V$ is the usual fluctuation operator describing interfacial static properties of this model (see, e.g., Ref. 3) with

$$
V=-\frac{1}{2} \xi^{-2}\left[1-3 \tanh ^{2}(z / 2 \xi)\right] \text {. }
$$

[In this convention negative, real $(-i \omega)$ corresponds to pure relaxational behavior.] All of the eigenfunctions and eigenvalues of $L_{z}$ are known and are provided in the background references. In this representation the function $\psi$ depends on $z$ only. The coefficient $\Gamma$ has been set to unity.

A systematic hydrodynamiclike analysis of (2.3) can be carried out ${ }^{22}$ built on lines introduced by Felderhof and Turski and Langer. ${ }^{7,8}$ Here solutions on either side of the interfacial region are matched, taking cognizance of the finite interfacial width. The unambiguous result, which may be reproduced in alternative fashion, including the methods presented here, is that in the hydrodynamic regime, $q \xi<1,(-i \omega) \sim-q^{3}$ for the relaxation of the so called capillary-wave-like excitations. On the other hand, it is possible to show from (2.3) that in the critical regime, $q \xi \gg 1,(-i \omega) \sim-q^{4}$ which, at this level of approximation, is just $\omega \sim q^{z}$ where $z=4$ is the bulk dynamic exponent describing relaxation of fluctuations in the homogeneous system at the critical point. ${ }^{21}$ The scaling function $\Omega$ in (1.1) contains a singularity reflecting this crossover even at this level of calculation.

A good approximation to the complete behavior of the dispersion relation can be constructed variationally as follows. From the equation of motion (2.3) one finds

$$
-i \omega=\min _{\phi}\left\{\frac{\langle\phi(-D+V) \phi\rangle}{\left\langle\phi D^{-1} \phi\right\rangle}\right\}
$$

as extremizing the right-hand side confirms. Here \langle\rangle represents the ordinary inner product. A good approximation should follow from the translation mode $\psi=\phi_{c}^{\prime}=d \phi_{c} / d z$. This mode, which generates the Goldstone mode corresponding to the broken translational invariance due to the establishment of the interface, is an exact solution as $q \rightarrow 0$. This mode is the dominant long-wavelength fluctuation, and effective interface models focus on it to the exclusion of all other modes of the system. Substituting this mode in the variational equation yields a dispersion relation of the form (1.1) with 
$z=4$ and the limits

$$
-i \omega \rightarrow\left\{\begin{array}{l}
-\Gamma \sigma q^{3} / 2 M^{2}, \quad q \xi \rightarrow 0 \\
-\Gamma q^{4}, \quad q \xi \rightarrow \infty .
\end{array}\right.
$$

In the present convention the minus means purely relaxational behavior. The two different limiting forms indicate that the scaling function $\Omega(x)$ displays the singular behavior $\Omega \sim 1 / x$ as $x \rightarrow 0$. Note the variational approximation correctly yields the limiting wave-number dependence and also correctly produces the constants of proportionality. $2 M$ is the bulk miscibility gap, and at this level the surface tension $\sigma$ is given by

$$
\sigma=\int\left[\phi_{c}^{\prime}(z)\right]^{2} d z
$$

The hydrodynamic limit agrees precisely with a completely macroscopic treatment of interfacial linear response based on the diffusion equation along with a local equilibrium assumption for the boundary condition at the interface (see, e.g., the work by Langer and Turski ${ }^{9}$ and Jasnow et al. ${ }^{23}$ with the nonequilibrium flux removed). The precise agreement of the variational approximation at both ends is very encouraging, and gives hope for its application to more complex (and more interesting) interface dynamics.

Some further insight into the working of the expression (2.5) can be obtained from the following perturbation analysis. Return to (2.3) which is rewritten for brevity as

$$
\Lambda \psi=A(B+\Delta) \psi
$$

with $\Lambda=i \omega$ and the eventual identifications for the operators $A=-D, B=-\partial_{z}^{2}+V(z)$, and $\Delta=\epsilon q^{2}$. The parameter $\epsilon$ is the expansion parameter, ${ }^{24}$ which ultimately gets set to unity. The logic here is that with $\epsilon=0$ the translation mode $\phi_{c}^{\prime}$ is an exact solution. In this formulation $A, B$, and $\Delta$ are Hermitian and the spectrum of $A$ is positive. Suppose one knows the solution of $A B \psi_{n}=\Lambda_{n} \psi_{n}$ and wants to solve $A(B+\Delta) \phi_{n}=E_{n} \phi_{n}$. Letting $\phi_{n}=\psi_{n}+\chi_{n}$ and $E_{n}=\Lambda_{n}+\delta_{n}$, one can proceed perturbatively. If one defines $\bar{\psi}$ through $\psi_{n} \equiv A \bar{\psi}_{n}$, one can show the orthogonality

$$
\left\langle\bar{\psi}_{m}, \psi_{n}\right\rangle \propto \delta_{m n}
$$

Then it is straightforward to find the leading-order result

$$
\delta_{n}=\frac{\left\langle\bar{\psi}_{n} A \Delta \psi_{n}\right\rangle}{\left\langle\bar{\psi}_{n} \psi_{n}\right\rangle}=\frac{\left\langle\psi_{n} \Delta \psi_{n}\right\rangle}{\left\langle\psi_{n} A^{-1} \psi_{n}\right\rangle}
$$

Taking $\psi_{n}=\phi_{c}^{\prime}(z)$ as noted above, one finds that the lowest-order perturbative result and the variational approximation yield the same expression for the eigenvalue and hence the interfacial dispersion relation.

One might question whether the limiting forms (2.6) are artifacts of the variational estimate or a first-order perturbation theory treatment. To increase our confidence in the validity of (2.6), we consider a simplified $U(\phi)$ with which we can solve for $\Omega$ exactly. Such model employs the "double Gaussian"

$$
U(\phi)= \begin{cases}\frac{1}{2} \kappa^{2}(\phi-M)^{2}, & \phi>0 \\ \frac{1}{2} \kappa^{2}(\phi+M)^{2}, & \phi<0 .\end{cases}
$$

This function is sometimes useful since the mathematics is greatly simplified. However, one cannot truly go towards the critical point from below, since in that limit the potential must go smoothly over to a quartic, as in Eq. (2.2). Nonetheless one may fix the correlation length $\xi=\kappa^{-1}$ and allow the wave number $q$ to vary from the hydrodynamic regime $q \xi<1$ over to "critical" with $q \xi \gg 1$.

The analysis for the system with an interface is straightforward. The classical solution or mean-field orderparameter profile has the form

$$
\phi_{c}(z)=M[1-\exp (-\kappa z)], \quad z>0
$$

with an odd continuation for negative $z$. To study fluctuations about the classical solution in linear response, one must solve Eq. (2.3) with

$$
V(z)=\kappa^{2}-2 \kappa \delta(z) \text {. }
$$

It is slightly easier to work with the "adjoint" $\bar{\psi}$ defined by $\psi=-D \bar{\psi}$, where the operator $D$ has been defined after Eq. (2.3). Now we seek solutions to

$$
(-D+V)(-D) \bar{\psi}=\Lambda \bar{\psi}
$$

with $\bar{\psi}$ continuous and with continuous first and second derivatives at $z=0$. The third derivative is discontinuous by the amount

$$
\bar{\psi}^{\prime \prime \prime}(0+)-\bar{\psi}^{\prime \prime \prime}(0-)=2 \kappa(-D) \bar{\psi}(0) \text {. }
$$

Writing $\Lambda=q^{4} \Omega(y)$, with $y=1 / 2 q^{2} \xi^{2}$, we find that $\Omega$ satisfies an algebraic equation,

$$
\begin{aligned}
\Omega^{4}-\left(2+2 y-y^{2}\right) \Omega^{3}+(1+ & \left.2 y-2 y^{2}-2 y^{3}\right) \Omega^{2} \\
& +2 y^{2}(1+y) \Omega+y^{4}=0 .
\end{aligned}
$$

It is tedious but straightforward to find the root [which also satisfies Eq. (2.15)]. Its limiting forms are $\Omega \rightarrow 1$ as $y \rightarrow 0$ ("criticality") and $\Omega \rightarrow(y / 2)^{1 / 2}$ as $y \rightarrow \infty$ (hydrodynamic regime). This exact result confirms (2.6) and supports the conclusion drawn from the approaches sketched above.

\section{MODEL $C$ INTERFACIAL DYNAMICS}

Model $C$ in the Hohenberg-Halperin ${ }^{21}$ scheme is defined by a nonconserved order parameter $\phi$ coupling to a conserved auxiliary (e.g., temperature) field $m$ according to

$$
\begin{aligned}
& \partial_{t} \phi=-\Gamma(\delta H / \delta \phi), \\
& \partial_{t} m=\lambda \nabla^{2}(\delta H / \delta m) .
\end{aligned}
$$

Here $H=H(\phi, m)=H(\phi)+H_{\times}(\phi, m)$, where $H(\phi)$ is just the usual Ginzburg-Landau expression given in (2.2), and the coupling is given by $H_{\times}=\frac{1}{2} \int[m-W(\phi)]^{2} d^{d} x$. 
$\Gamma$ and $\lambda$ are the kinetic coefficients. A convenient choice of $W$ is $W(\phi)=\frac{1}{2} w \phi^{2}$, which we adopt. Straightforward analysis shows that the equilibrium interface $\phi_{c}$ has the same hyperbolic tangent form as discussed above, and the corresponding auxiliary field is given by $m_{c}=W\left(\phi_{c}\right)$. When the equations are linearized about the equilibrium interface, one is faced with a $2 \times 2$ eigenvalue problem of the form

$$
\partial_{t}\left[\begin{array}{c}
\delta \phi \\
\delta m
\end{array}\right]=\mathbf{D L}\left(\begin{array}{c}
\delta \phi \\
\delta m
\end{array}\right]
$$

where

$$
\mathbf{D}=\left(\begin{array}{cc}
-\Gamma & 0 \\
0 & \lambda \nabla^{2}
\end{array}\right), \quad \mathbf{L}=\left(\begin{array}{cc}
-\nabla^{2}+U_{c}^{\prime}+\left(W_{c}^{\prime}\right)^{2} & -W_{c}^{\prime} \\
-W_{c}^{\prime} & 1
\end{array}\right)
$$

The subscript $c$ indicates the functions are evaluated at $\phi_{c}$.

At criticality the modes decouple with respective frequencies $-\Gamma k^{2}$ and $-\lambda k^{2}$. Below $T_{c}$ deep in the ordered phase, the $\phi$-like mode decays rapidly for all $k$ whereas the $m$-like (temperature) mode decays proportionally to $-k^{2}$ with a modified coefficient. Around an interface solution $\phi_{c}$, however, we resort to the variational estimate

$$
-i \omega) \leq-\frac{\langle\boldsymbol{\Psi} \mathbf{L} \boldsymbol{\Psi}\rangle}{\left\langle\boldsymbol{\Psi} \mathbf{D}^{-1} \boldsymbol{\Psi}\right\rangle},
$$

where $\boldsymbol{\Psi}$ is a two-component vector as in (3.2). The translation mode now can be shown to be $\boldsymbol{\Psi}=\left(\phi_{c}^{\prime}, m_{c}^{\prime}\right)$, which is an exact solution to the equations of motion with zero frequency for the case $q=0$. Using this $\Psi$ in (3.4), which is analogous to the choice in Sec. II, one finds an estimate for the coupled mode frequency

$$
-i \omega \simeq-\Gamma q^{2}\left(1+\frac{\Gamma w^{2}}{\lambda} g(q \xi)\right)
$$

Far from $T_{c}, g \rightarrow 1$, while as $q \xi \rightarrow \infty, g \rightarrow 0$. The kinetic coefficient for this coupled interfacial mode crosses over from $\Gamma /\left(1+\Gamma w^{2} / \lambda\right)$ in the ordered phase to $\Gamma$ as $T \rightarrow T_{c}-$.

\section{CONCLUDING REMARKS}

In this short paper we have drawn together some of the threads concerning interfacial dynamics in systems with a conserved order parameter (model $B$ ) near equilibrium. We have shown that a variational approximation (and also systematic perturbation theory) can be used to interpolate the dispersion relation between the hydrodynamic $(q \xi<<1)$ and critical $(q \xi>1)$ regimes. In the latter regime the interplay between bulk and interfacial modes becomes important. This interpolation allows extension, if not truly to the critical point, where fluctuation analysis beyond the mean-field dynamical treatment is required, at least to the regime of thick walls. A simplified model, in which the effective potential $U(\phi)$ is replaced by a double Gaussian, is solved exactly yielding an explicit form for the crossover function in the interfacial dispersion relation, $\Omega(q \xi)$. The limiting forms are in agreement with the variational and perturbative approaches discussed in Sec. II.

We have also considered the case of a conserved auxiliary (temperature) field coupling to a nonconserved order parameter (model $C$ ). A variational approximation can be constructed which shows the crossover of the effective kinetic coefficient for the interfacial modes. Far from $T_{c}$ the coupling to the slow mode affects the decay of interface fluctuations, but nearer criticality the modes effectively decouple. (Although we did not analyze model $D$ explicitly, we would surmise that similar conclusions would result using similar techniques.) For both models considered here, Janssen et al. ${ }^{25}$ have also investigated the exact eigenvalues for dynamic equations arising from "simplified" potentials like double and triple parabolas. However, the question of crossover was not addressed.

We comment here briefly on some interface equations of motion introduced by Ohta and Kawasaki. ${ }^{18-20}$ Such equations were advanced to describe the hydrodynamic regime (of long-wavelength interface variations compared to interface thickness) and may be used in a variety of nonequilibrium situations. Their main virtue is their ability to deal with the nonlinear regime in which interfacial distortions have finite amplitude (comparable to or greater than the wavelength). They may also be applied near equilibrium, and the discussion is restricted to that case. The interface equations have a close resemblance to the variational and perturbative schemes sketched in Sec. II. Since the approximate schemes have been shown to interpolate correctly to the critical regime, it seems as though the Kawaski-Ohta interface equations (at least in the linear regime) can also be pushed toward criticality (or at least to a domain in which the walls can be considered thick). Of course, the true interest is in the nonlinear regime, and further work is necessary to determine whether the equations are restricted to describe walls thin compared to the scale of interface undulations. Such questions are particularly interesting in considering driven systems.

The approaches and results discussed here give some confidence that they can be extended to more complex and interesting cases. It is hoped that work on fluids can be reported in the near future.

\section{ACKNOWLEDGMENTS}

One of us (D.J.) wishes to thank Dr. Bernd Schaub for helpful discussions during the early stages of this work and Professor Joseph Rudnick for his interest and suggestions. The authors are grateful to the National Science Foundation through the Division of Materials Research for supporting this work under Grants No. DMR-8302326, No. DMR-86-13030, and No. DMR-85-04716. 
${ }^{1}$ J. W. Gibbs, Collected Works (Longmans, Green, New York, 1928), Vol. 1, p. 55.

${ }^{2}$ B. W. Widom and J. S. Rowlinson, Molecular Theory of Capillarity (Clarendon, Oxford, 1982).

${ }^{3}$ D. Jasnow, Rep. Prog. Phys. 47, 1059 (1984).

${ }^{4}$ D. Jasnow, in Phase Transitions and Critical Phenomena, edited by C. Domb and J. L. Lebowitz (Academic, New York, 1986), Vol. 10.

${ }^{5}$ L. D. Landau and E. M. Lifshitz, Fluid Mechanics (Pergamon, New York, 1959).

${ }^{6}$ G. Saffman and G. I. Taylor, Proc. R. Soc. London, Ser. A 245, 312 (1958).

${ }^{7}$ B. U. Felderhof, Physica (Utrecht) 48, 541 (1970).

${ }^{8}$ L. A. Turski and J. S. Langer, Phys. Rev. A 22, 2189 (1977).

${ }^{9}$ J. S. Langer and L. A. Turski, Acta. Metall. 25, 1113 (1977).

${ }^{10}$ K. A. Jackson and J. D. Hunt, Acta. Metall. 13, 1215 (1965).

${ }^{11}$ S. C. Huang and M. E. Glicksman, Acta Metall. 29, 701 (1981); 29, 717 (1981).

12J. W. Cahn and S. M. Allen, J. Phys. (Paris), Colloq. 38, C7-
51 (1977).

${ }^{13}$ S. M. Allen and J. W. Cahn, Acta. Metall. 27, 1085 (1979).

${ }^{14}$ J. S. Langer, Rev. Mod. Phys. 52, 1 (1980).

${ }^{15}$ R. Bausch, V. Dohm, H. Janssen, and R. K. P. Zia, Phys. Rev. Lett. 47, 1837 (1981).

${ }^{16}$ G. Jug and D. Jasnow, Phys. Rev. B 30, 6795 (1984).

${ }^{17}$ G. Jug and D. Jasnow, Phys. Rev. B 31, 7385 (1985).

${ }^{18}$ K. Kawasaki and T. Ohta, Prog. Theor. Phys. 67, 147 (1982).

${ }^{19}$ K. Kawasaki and T. Ohta, Physica 118A, 175 (1983).

${ }^{20}$ T. Ohta, Ann. Phys. (N.Y.) 158, 31 (1984).

${ }^{21}$ P. C. Hohenberg and B. I. Halperin, Rev. Mod. Phys. 49, 435 (1977).

${ }^{22}$ D. Jasnow (unpublished).

${ }^{23}$ D. Jasnow, D. A. Nicole, and T. Ohta, Phys. Rev. A 23, 3192 (1981).

${ }^{24}$ D. M. Kroll (unpublished).

${ }^{25}$ H. K. Janssen, R. Bausch, V. Dohm, and R. K. P. Zia (unpublished). 